\title{
NOTES
}

\section{Specific Polymerization Behavior of Triallyl Isocyanurate. Accumulation of Radicals before Gelation}

\author{
Akira Matsumoto, ${ }^{\dagger}$ Daisuke Ishinara, Kazuyoshi Nishizawa, Hiroyuki Aota, \\ Yoshihiro TaKaYama, ${ }^{*}$ Akinori KamEYama, ${ }^{*}$ and Tsuneo NAKANISHI* \\ Department of Applied Chemistry, Faculty of Engineering and High Technology \\ Research Center, Kansai University, Suita, Osaka 564-8680, Japan \\ * Nippon Kasei Chemical Co., Ltd., 34 Takayama, Onahama, Iwaki-shi, \\ Fukushima 971-8101, Japan
}

(Received June 17, 1999)

KEY WORDS Triallyl Isocyanurate / Allyl Polymerization / Accumulated Radical / Gelation /

As part of our continuing studies concerned with free-radical crosslinking polymerization of multivinyl compounds, the previous article dealt with the inhomogeneity of network structure accompanied by radical accumulation in the polymerization of multiallyl compounds including triallyl isocyanurate (TAIC), triallyl cyanurate (TAC), diallyl phthalate, diallyl isophthalate, diallyl terephthalate, and triallyl trimellitate. ${ }^{1}$ Pendant allyl radicals belonging to the densely crosslinked part were detected by ESR spectroscopy and conversion dependence of the pendant allyl radical accumulation was different for each polymerization. These matters are discussed in relation to differences of the network formation, especially the occurrence of intramolecular crosslinking leading to inhomogeneity of network structure.

The polymerization of TAIC was specific as compared with other multiallyl compounds. In TAIC polymerization radical accumulation was remarkably observed even at an early stage of polymerization without gelation, whereas accumulated radicals were detected only beyond the gel points for the polymerizations of other multiallyl compounds. This prompted us to explore in more detail the accumulation of radical with conversion in the polymerization of TAIC.

\section{EXPERIMENTAL}

TAIC, TAC, allyl dipropyl isocyanurate (ADPIC), and allyl diisopropyl isocyanurate (ADiPIC) were supplied by Nippon Kasei Chemical Co., Ltd. These monomers were purified by vacuum distillation under nitrogen. 2,2'-Azobisisobutyronitrile (AIBN) as initiator and benzene as solvent were purified by conventional methods.

Polymerization and X-band ESR measurement were carried out as described previously. ${ }^{1,2}$ The radical concentration of polymerization was determined by comparing with an external standard $\mathrm{Mn}^{2+}$ (JEOL ES-DM1).

\footnotetext{
To whom correspondence should be addressed.
}

\section{RESULTS AND DISCUSSION}

Figure 1 shows the conversion-time curves for bulk copolymerization of TAIC with its isomer TAC using $0.05 \mathrm{~mol} \mathrm{L^{-1 }}$ of AIBN at $60^{\circ} \mathrm{C}$. The rate of polymerization decreased and the gel-point conversion increased linearly with the amount of TAC in the feed. Figure 2 illustrates the variation of ESR spectra of accumulated radical with time for the polymerization of TAIC as a typical example. Absolute radical concentrations were determined by calibrating with 4-hydroxy2,2,6,6-tetramethylpiperidine-1-oxyl dissolved in benzene.

Figure 3 demonstrates the accumulation of radicals with the progress of polymerization. The concentration of accumulated radical decreased with added TAC. TAIC was then copolymerized with allyl acetate (AAc) as a sterically unhindered allyl compound. The radical accumulation drastically decreased by the addition of AAc and eventually no radical was detected for TAIC/AAc $(50 / 50 \mathrm{~mol} / \mathrm{mol})$ copolymerization. Similar

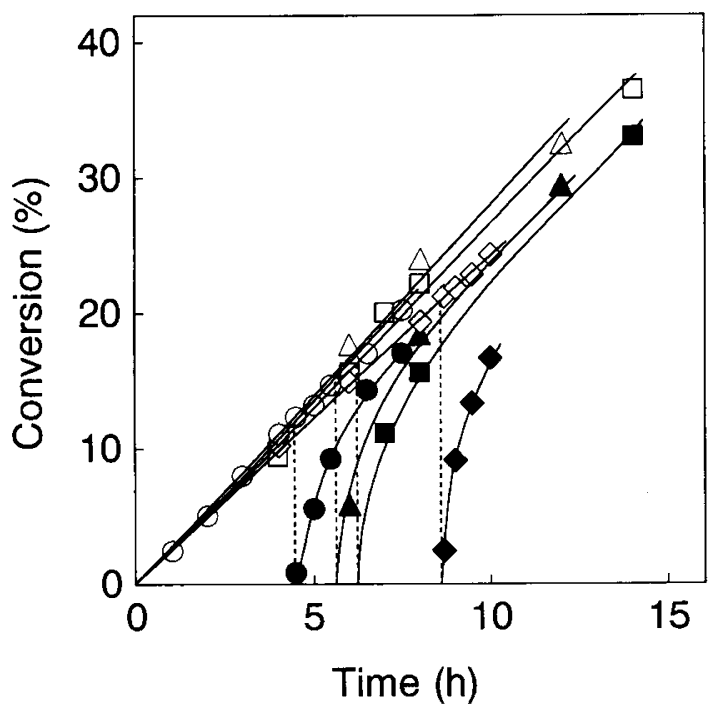

Figure 1. Conversion-time curves for $(O, \odot)$ TAIC and $(\diamond, \diamond)$ TAC homopolymerizations and TAIC/TAC $((\triangle, \Delta) 80 / 20$ and $(\square$, D) $50 / 50 \mathrm{~mol} / \mathrm{mol}$ ) copolymerizations. Polymerization was conducted in bulk using $0.05 \mathrm{~mol} \mathrm{~L}^{-1}$ of AIBN at $60^{\circ} \mathrm{C}$. Open and full symbols correspond to total and gel polymers, respectively. 


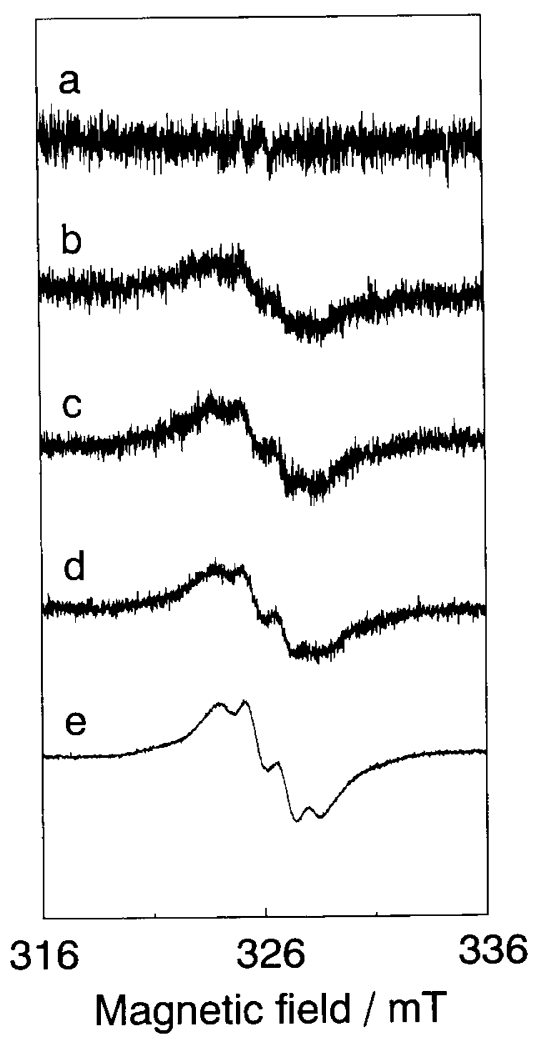

Figure 2. Variation in ESR spectra of accumulated radicals with time for the polymerization of TAIC (see Figure 1): (a) before polymerization; (b) $2 \mathrm{~h}$; (c) $4 \mathrm{~h}$; (d) $6 \mathrm{~h}$; (e) $24 \mathrm{~h}$ (completely cured).

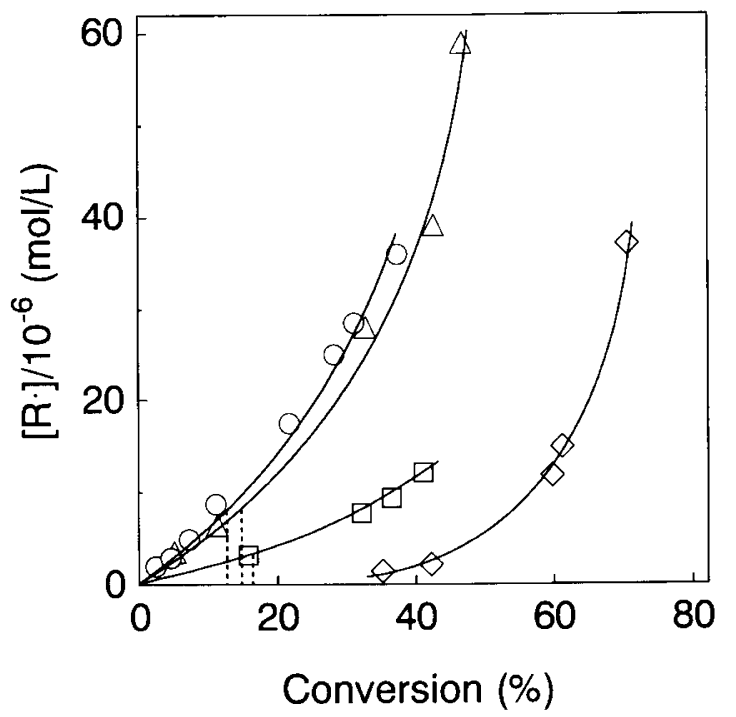

Figure 3. Accumulation of radicals with progress of polymerization (see Figure 1).

results were observed for the copolymerization of TAIC with vinyl acetate. The accumulation of radicals was enhanced by the copolymerization of TAIC with ADPIC or ADiPIC as a monoene counterpart of TAIC (Figure 4), although the shape of spectrum did not change by the addition of ADPIC or ADiPIC. No radical accumulation was observed in the homopolymerization of ADPIC or ADiPIC. TAC was copolymerized with ADiPIC. A rather suppressed radical accumulation by the addition of ADiPIC was observed after gelation.

These results are in conformity with our previous

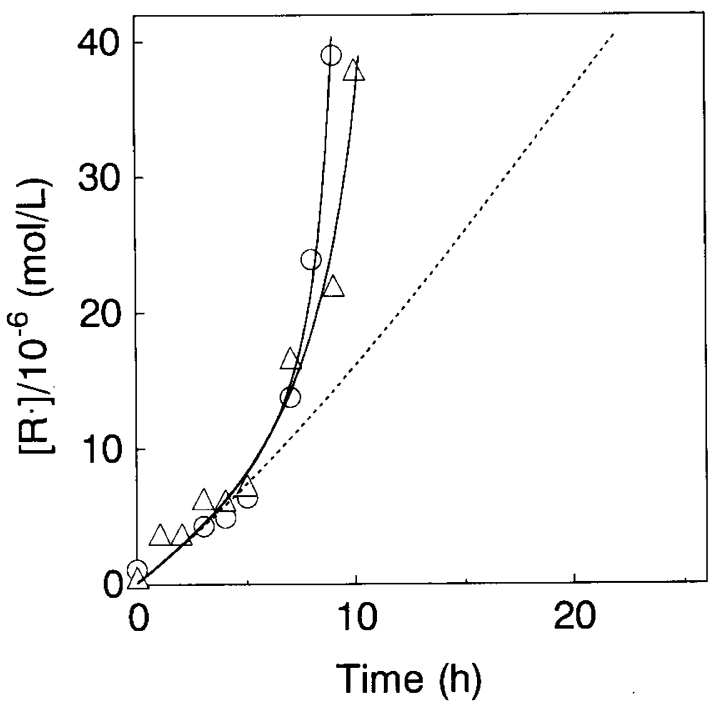

Figure 4. Accumulation of radicals with progress of polymerization in (O) TAIC/ADPIC and $(\triangle)$ TAIC/ADiPIC $(2 / 1 \mathrm{~mol} / \mathrm{mol}) \mathrm{co}-$ polymerizations. Dotted line corresponds to TAIC homopolymerization as a comparison.

speculation that the pendant allyl radical may be detected by ESR. ${ }^{1}$ That is, there may be four radicals in the polymerization system, a primary radical, growing polymer radical, monomeric allyl radical and polymeric pendant allyl radical. First, no substantial difference was observed in the use of either AIBN or BPO initiator, indicating that accumulated radicals are not primary radicals. Secondly, no radical detection appeared for ADPIC and ADiPIC polymerization, suggesting that growing polymer radicals are not assigned to accumulated radicals. Thirdly, no possibility of assignment to monomeric allyl radicals was supported by the fact that no accumulated radicals were observed in the copolymerization of TAIC with AAc where monomeric allyl radicals would be always generated.

We may discuss briefly the accumulation of radicals in connection with the specific polymerization behavior of TAIC ${ }^{3,4}$ : The polymeric pendant allyl radicals would be formed as degradative chain transfer, ${ }^{5}$ i.e., chain transfer of the growing polymer radical to the pendant allyl groups of prepolymer, characteristic of the polymerization of allyl compounds. The chain transfer reaction may occur through: 1) intramolecular consecutive or non-consecutive cyclization reaction with allyl groups in the same primary polymer chain as a terminal or nonterminal unit including penultimate, penpenultimate, and so on, and 2) intermolecular or intramolecular crosslinking reactions with pendant allyl groups of another or the same prepolymer. The former intramolecular cyclization would be excluded considering the following facts. That is, intramolecular cyclization is rather complicated in the polymerization of TAIC, i.e., it occurs not only in the same monomer unit as a consecutive cyclization, but also with the pendant allyl groups belonging to nonterminal units as a non-consecutive cyclization leading to a larger ring formation as expected from the molecular model, although nonconsecutive addition occurred significantly in the polymerizations of diallyl terephthalate ${ }^{6,7}$ and triallyl citrate. ${ }^{8}$ In this connection, the solution polymerization of TAIC 


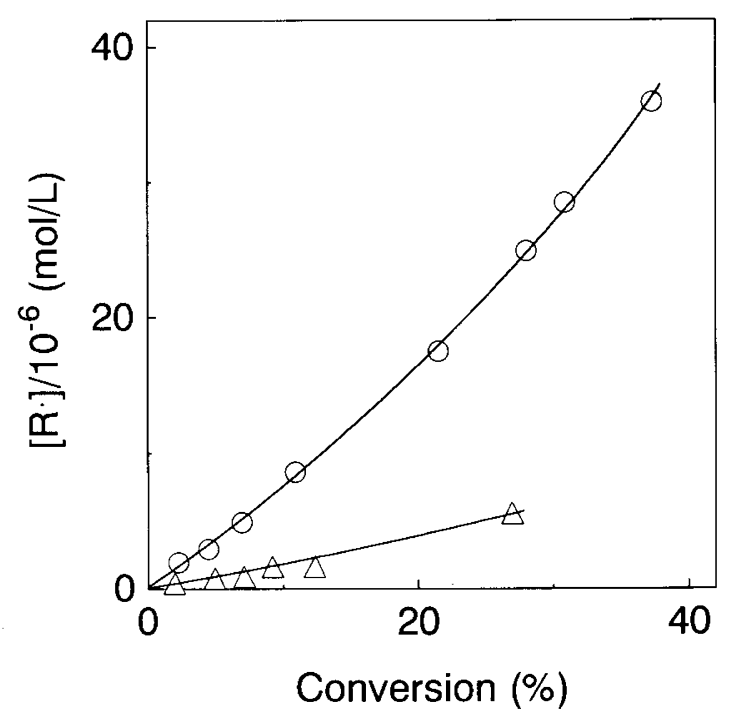

Figure 5. Accumulation of radicals with progress of polymerization in $(O)$ bulk and $(\triangle)$ solution polymerizations in benzene at a dilution of $1 / 5$.

was conducted in benzene at a dilution of $1 / 5$. As is shown in Figure 5, the concentration of accumulated radicals was quite reduced in solution polymerization, indicating that the radical accumulation is not ascribed to the intramolecular cyclization.

Finally, we discuss the enhanced accumulation of radicals by the copolymerization of TAIC with ADPIC or ADiPIC. The copolymerization with ADPIC or ADiPIC, a compound in which two allyl groups of TAIC are saturated, corresponds to the forced introduction of the bulky, noncyclic TAIC units into the polymer chain. The resulting precopolymer chain may thus have less flexibility, probably leading to a formation of more stable pendant allyl radical.

A detailed picture is presented for a specific polymerization of TAIC accompanied by remarkable accumulation of radicals even at an early stage without gelation.

\section{REFERENCES}

I. H. Aota, T. Kishimoto, A. Matsumoto, and S. Matsunami, $J$. Net. Polym. Jpn., 18, 1 (1997).

2. H. Aota, Y. Sanai, A. Matsumoto, and M. Kamachi, Polym. J., 28, 867 (1996).

3. A. Matsumoto, F. Hirai, Y. Sumiyama, H. Aota, Y. Takayama, A. Kameyama, and T. Nakanishi, Eur. Polym. J., 35, 195 (1999).

4. A. Matsumoto, T. Kubo, K. Watanabe, H. Aota, Y. Takayama, A. Kameyama, and T. Nakanishi, Eur. Polym. J., in press.

5. P. D. Bartlett and R. Altschul, J. Am. Chem. Soc., 67, 812, 816 (1945).

6. A. Matsumoto and M. Oiwa, Nippon Kagaku Zasshi, 90, 1278 (1969).

7. A. Matsumoto, H. Sasaki, and M. Oiwa, Makromol. Chem., 166, 179 (1973).

8. A. Matsumoto, T. Ohata, and M. Oiwa, Bull.Chem. Soc. Jpn., 47, 673 (1974). 